\title{
Fast Domain Growth through Density-Dependent Diffusion in a Driven Lattice Gas
}

\author{
Lisa K. Wickham, and James P. Sethna \\ Laboratory of Atomic and Solid-State Physics, \\ Cornell University, \\ Ithaca, NY 14853-2501.
}

\begin{abstract}
We study electromigration in a driven diffusive lattice gas (DDLG) whose continuous Monte Carlo dynamics generate higher particle mobility in areas with lower particle density. At low vacancy concentrations and low temperatures, vacancy domains tend to be faceted: the external driving force causes large domains to move much more quickly than small ones, producing exponential domain growth. At higher vacancy concentrations and temperatures, even small domains have rough boundaries: velocity differences between domains are smaller, and modest simulation times produce an average domain length scale which roughly follows $L \sim t^{\zeta}$, where $\zeta$ varies from roughly .55 at $50 \%$ filling to roughly .75 at $70 \%$ filling. This growth is faster than the $t^{1 / 3}$ behavior of a standard conserved order parameter Ising model. Some runs may be approaching a scaling regime. A simple scaling picture which neglects velocity fluctuations, but includes the cluster size dependence of the velocity, predicts growth with $L \sim t^{1 / 2}$. At low fields and early times, fast growth is delayed until the characteristic domain size reaches a crossover length which follows $L_{\text {cross }} \propto E^{-\beta}$. Rough numerical estimates give $\beta=.37$ and simple theoretical arguments give $\beta=1 / 3$. Our conclusion that small driving forces can significantly enhance coarsening may be relevant to the $\mathrm{YB}_{2} \mathrm{Cu}_{3} \mathrm{O}_{7-\delta}$ electromigration experiments of Moeckly et al. [13].
\end{abstract}

Typeset using REVTEX 


\section{INTRODUCTION}

The study of spinodal decomposition and coarsening in quenched Ising models has been vigorously pursued [1]. Binder and Stauffer [2] predicted that, following a quench at $t=0$, the structure function of a coarsening system would grow with a single length scale $L(t)$. Numerical studies have verified that, when this length scale is removed from the results, the reduced structure factor is very nearly constant in time [3]. Lifshitz and Slyozov [4] gave a further prediction: domain size should asymptotically grow as $L \sim t^{1 / 3}$ for a conserved order parameter (COP) model. Monte Carlo simulations [5] have checked this result. All of this theory describes the equilibrium case, where nothing acts on the coarsening process besides a thermal bath. In real systems, however, phase segregation can be affected by several influences, including gravity, elastic stress, or electric fields. Such forces often push material around, instead of preferring one phase over another. Given this wide area of potential experimental application, it seems reasonable to ask: what happens when you take a COP Ising model and apply a uniform force to push particles (up spins) across the lattice?

This type of model was first introduced by Katz, Lebowitz, and Spohn [6], who found that the external driving force raised $T_{c}$. Subsequent research has carefully investigated the ordering phase transition of this model. [7] In addition to work which analyzed interface roughness [8] and domain shape [9], one study has checked to see whether the scaling and growth law results of the equilibrium model can carry over to the nonequilibrium one [10]. Such studies of the driven diffusive lattice gas (DDLG) have almost always employed the same Monte Carlo dynamics — those of Kawasaki [11]. For this specific class of model, there is no barrier to hinder particle motion along the interface between two phases, and domains tend to elongate along the direction of the force. When considering a directionally averaged measure of domain size, however, Yeung et al. [10]. found that the Kawasaki form of the DDLG showed familiar $L_{a v} \sim t^{1 / 3}$ coarsening behavior.

The present study finds that this slow rate of coarsening, as well as the orientation of domains, is model-dependent. Noting that nonequilibrium problems have an inherent 
sensitivity to dynamics, we have studied a DDLG with particle mobility that goes down when the number of bonds to neighbors goes up. The resulting motion has free diffusion of single particles across empty spaces. (A similar bond-counting approach was used to model electromigration of thin films on semiconductors [12], but that work did not study coarsening.) In our model, the external driving force bunches domains up along the field (so that they lengthen in the transverse direction), and it can push entire domains of vacancies across the lattice so that they sweep up other vacancies and grow quickly. For moderate lattice fillings, the resulting domain radius grows as $L_{a v} \sim t^{\zeta}$, where $\zeta$ varies roughly from 0.4 to 0.7 . For high concentrations of particles, the early stages of growth can be exponential in time.

When we approached the problem of coarsening in an electric field, we were interested in fast motion of isolated particles through the middle of a domain, rather than along an interface. Such bulk diffusion was relevant to the electromigration studies of Moeckly, Lathrop, and Buhrman [13]. In their room temperature observations of $\mathrm{YB}_{2} \mathrm{Cu}_{3} \mathrm{O}_{7-\delta}$ thin film devices, they found that a small electric bias $\left(\sim 10^{3} \mathrm{~V} / \mathrm{cm}\right)$ could produce macroscopic motion of oxygen. The associated force was so tiny that it would only have moved an oxygen atom a few lattice constants per second in a fully oxygenated sample, where the activation energy for oxygen motion is about $1 \mathrm{eV}$ [14] and the diffusion constant is about $10^{-12} \mathrm{~cm}^{2} / \mathrm{s}$ near room temperature [15]. In an oxygen depleted region, however, small forces may have a large impact: internal friction measurements give an activation energy of $0.1 \mathrm{eV}$ for motion of a completely isolated oxygen atom, and the chemical diffusion data of LaGraff et al. [16] suggest that the diffusion constant of YBCO can rise by more than an order of magnitude as the oxygen in the chain plains is depleted.

To study the effects of such differences in mobility, we wanted the simplest model that could describe a density-dependent diffusion constant. We therefore chose a two dimensional DDLG with modified continuous Monte Carlo dynamics [5]. Thus, we group atoms according to their coordination $q$, increment time by an amount which increases as the number of highly 
mobile atoms decreases, and propose a move from list $q$ with probability

$$
P[q]=d t(4-q) N(q) e^{-4 J q}
$$

where $N[q]$ is the number of $q$-coordinated atoms. This continuous Monte Carlo scheme satisfies detailed balance, so the equilibrium state at $\Delta=0$ is that of the nearest-neighbor Ising model: $\mathcal{H}=-J \sum_{\langle i j\rangle} S_{i} S_{j}$. This dynamics allows atoms with low coordination to move quickly. It also produces a basic particle-hole asymmetry, illustrated by the fact that isolated atoms can zip across vacant spaces (rate 1), while isolated holes hardly move $\left(\right.$ rate $\left.e^{-12 J}\right)$. We include the electric potential by accepting all proposed forward moves, a fraction $e^{-\Delta}$ of the proposed sideways moves, and only $e^{-2 \Delta}$ of the proposed moves against the field, where $2 \Delta k T$ is a local potential difference along the field [17]. Motivated by the YBCO experiments, we have focused much of our attention on the limits of high particle concentration, relatively low fields, and strong coupling to nearest neighbors (i.e. a highly concentration-dependent mobility).

Figure 1 shows two of the interesting behaviors we found. In both pictures, the black regions are vacancy clusters which move collectively downwards as an external force pushes (white) particles up. In the symmetry-breaking field, the vacancy blocks become short and wide [18]. The pictures on the left (Fig. 1a and 1b) have 90\% lattice filling. Here, isolated runaway processes dominate: large domains move much faster than small ones and sweep up many vacancies, thus becoming even bigger and faster. We discuss such processes in the following section on exponential growth. The pictures on the right have $70 \%$ lattice filling. Here, blocks of all length scales are moving and combining, and a mean domain radius grows as a power in time. Note that the late snapshot at the bottom (Fig. 1d) resembles a scaledup version of the snapshot at the top (Fig. 1c). In section III, we evaluate scaling collapses and we construct a simple picture for domain growth in this regime. Finally, in section IV, we will look at small driving forces. In this limit, we find that the early stages of growth show the $L \sim t^{1 / 3}$ behavior expected for the zero field case, and then a crossover to fast growth occurs. We interpret this crossover as the time at which the area swept out through 
linear motion along the field equals the area visited by diffusive motion, and we derive the field dependence of the domain size at crossover.

\section{EXPONENTIAL GROWTH}

The runaway growth of the high filling regime is fundamentally tied to a separation of time-scales produced by faceting. The pictures in Figure 1 were generated with strong coupling between neighbors, so atoms with two neighbors moved much more quickly than atoms with three. In this regime, the base of each vacancy domain tends to be flat, with all atoms having three neighbors. After a stagnant period, one of these strongly pinned atoms pops out of the base and leaves behind two doubly coordinated particles. The remaining atoms then have a lower barrier to motion. One by one, the rest of the row soon dislodges and moves rapidly across the empty space. Under such conditions, one would expect the velocity of a region to be proportional to its horizontal width (i.e. the number of ways to produce the initial break). Figure 3 shows this behavior at low temperatures for isolated vacancy domains. Periodically, a domain will collide with a vacancy in its path and that will provide the initial break to move the domain through an extra row of atoms. Again, the rate of such motion increases linearly with the width of the domain.

Domain size is therefore a crucial factor in determining growth. Besides moving more quickly to sweep up new vacancies, wide blocks clear larger regions as they move. In general, we expect:

$$
\frac{d n}{d t}=w \cdot \Delta v \cdot c .
$$

Here, $n$ is the area of the block in question, $w$ is its width, $c$ is the concentration of vacancies in the region ahead of it, and $\Delta v$ is the relative velocity of the block we are describing (in comparison to that of vacancies which it overtakes). For low temperatures and low vacancy concentrations, small vacancy blocks will move at negligible velocities and large blocks will move with $v \sim w$. In this regime we expect:

$$
\frac{d n}{d t} \propto w \cdot w \cdot c .
$$


If the width and height of a region scale similarly, then the above result gives: $d n / d t \propto n$ or $n$ growing exponentially in time. In practice, we find that width grows more quickly than height. This tendency should only enhance the rate of growth.

To check this prediction against our simulation, we calculated two-point correlation functions in both horizontal and vertical directions. For a rough measure of length-scales, we used the width at quarter max of each of our correlation functions [19]. Figure 2 shows vertical block size, horizontal block size, and the product of the two (a typical domain area) as a function of time. To run the simulation efficiently enough to observe a large range of size, we used a fast model with nearly infinite coupling (where uncoordinated atoms always moved first, and then all singly coordinated atoms moved). As figure 1 demonstrates, we found the same behavior at low temperature for standard finite-coupling dynamics. Note that the vertical scale on these plots is logarithmic, so the straight line observed does indicate exponential growth.

Notice that the runaway growth does not continue indefinitely. For very large domains, the time required to move a full row of atoms from bottom to top is comparable to the time between initial "three-moves". If the rate of $q=2$ moves is the limiting step, then motion from each kink in the domain can proceed independently and large blocks will approach a terminal velocity. Crossover to this behavior will occur when the time required to move an entire row of atoms through sequential $q=2$ moves is approximately equal to the expected waiting time before one atom in that row moves from a triply coordinated site. The slowdown in growth in figure 2 occurs when these two time-scales are comparable to each other. Figure [3 shows the velocity of a vacancy domain in an empty lattice as a function of domain width at two different temperatures. Note that the low temperature plot is fairly linear, while the high temperature results do indeed approach a terminal velocity. Figure 6 shows simulation results with a domain roughening crossover which varies with temperature.

There is another way to produce rough domain bases and eliminate exponential growth. In the case where vacancy clusters are constantly running into one another, their bases will always contain doubly coordinated atoms, and $q=2$ moves will again be the rate 
limiting step. Figure 5 shows the changeover from exponential to power-law growth as the number of vacancies increases. Looking at configurations with $82 \%$ filling, we see isolated runaway domains whose acceleration slows as their bases become rough. This behavior makes intuitive sense because, in runaway growth, the size of large domains increases more quickly than the spacing between small ones, so large domains can grow to be larger than a typical interdomain spacing. We will find later that horizontal correlation functions in the power-law growth regime nearly scale, so the relationship between domain width and horizontal domain spacing remains nearly fixed. This is consistent with our observation that growth which starts in the crowded, power-law regime tends to stay power-law.

\section{POWER-LAW GROWTH AT LOWER FILLINGS}

We have found that the exponential growth regime occurs for low temperatures and low vacancy concentrations, where only a few domains become large enough to respond strongly to the external field. At lower particle fillings, most vacancies will join clumps soon after coarsening begins, since most of the vacancies are connected through atoms with single or double coordination at quench. At such fillings, vacancy domains no longer move through a nearly stationary sprinkling of tiny vacancy clumps. Instead, the lattice contains a distribution of block sizes, most of which are moving steadily in the field. Frequent collisions between domains provide sources of fast moving atoms, so that motion is not characterized by long waiting times with flat domain bases. Thus, we no longer expect the velocity of a domain to be proportional to its width.

Figures 6 and 7 show results from the simulation at lower fillings. The first, a check

for dynamical scaling, gives clear evidence that domains of all length scales are growing at similar rates. The horizontal correlation function shows strong hints of scaling, but the vertical correlation function has an anticorrelation dip which grows more pronounced with time. (That is, the regions between vacancy domains are becoming more thoroughly swept out.) Although growth in this regime is not completely self-similar, a scaling picture 
may be a useful first step towards describing coarsening at these fillings. Figure 7 shows characteristic domain area as a function of time for $60 \%, 70 \%$, and $80 \%$ concentrations. This growth is significantly faster than the $t^{1 / 3}$ behavior of a zero field model. If we fit growth at each concentration to domain area $\sim t^{2 \zeta}, \zeta$ varies from about .65 at $60 \%$ filling to approximately .75 at $70 \%$ and $80 \%$ filling.

Although the behavior of our model in this moderately full regime is complex, we have tried to piece together a simple picture which would mimic the observations described above. We start with the question: in a scaling regime where growth is still dominated by catchup events, what kind of velocity distribution would produce linear domain growth? An elementary argument proceeds as follows: we can describe each time in a scaling regime with characteristic horizontal and vertical length-scales $L_{h}$ and a $L_{v}$. In a typical collision, the area gained by a vacancy cluster will scale with the product of these two lengths, i.e.

$$
d n \sim L_{h} \cdot L_{v}
$$

A typical time between collisions will scale as the vertical length-scale divided by the velocity difference of the two colliding domains:

$$
d t \sim L_{v} / \Delta v
$$

Together, these two results indicate that the area of a typical domain will increase linearly in time if $\Delta v \sim 1 / L_{h}$.

Is this scaling picture useful for understanding simulation results? One obvious objection is that vertical correlations in our model do not settle into a final scaling shape until late in the simulation, and so the typical vertical spacing between domains does not scale perfectly with the vertical height of the domains. Also, we have neglected any enhancement in coarsening due to velocity fluctuations, and in fact our simulation results indicate that domain areas in this regime have somewhat faster than linear growth in time. Realizing that our scaling picture is an approximate description, at best, we have investigated the size dependence of domain velocities. Recall that a large domain with several kinks in its base 
should approach a terminal velocity where motion proceeds from each kink independently. Is the dominant correction to this terminal velocity a term of the form $v_{c o r} / L_{h}$ ? Note that this the form one might expect if the dominant correction is due to behavior at the sides of a domain base. Figure 8 shows velocity plotted against $1 / w$ for domains of various size moving through empty space at high temperature, so that the domain bases had several kinks. We tried plotting velocity vs. $w^{x}$ and find the best asymptotic linear fit for $x=.7$ to 1.2. Figure 9 shows velocity as a function of $1 / w$ in an actual simulation run at $70 \%$ filling. Despite poor statistics in the latter plot, figures 8 and 9 together seem to confirm that vacancy clusters in our model approach constant velocity with $1 / L_{h}$ corrections. Thus, our scaling picture may provide a first step towards explaining observed growth at these fillings.

For fillings below $50 \%$, we must focus on domains of particles, instead of vacancies. These clumps of particles actually move against the field direction while a wind of particles sweeps into them on one side and tears particles away on the other. Figure 10 shows preliminary simulation results at these fillings. Domain area grows roughly as $t^{.75}$ at $20 \%$ filling $(\zeta \approx .4)$, as $t^{.95}$ at $30 \%$ filling $(\zeta \approx .5)$, and as $t^{1.1}$ at $50 \%$ filling $(\zeta \approx .55)$. Note that growth at low fillings is dominated by the shorter time-scales associated with motion of atoms with few neighbors. Note also that the growth exponent $\zeta$ increases with filling. We do not at present have an explanation for the latter effect.

\section{LOW FIELD CROSSOVER}

For high fillings and strong interparticle couplings, we have seen that large external fields can dramatically enhance coarsening. In most experimental applications, however, the potential difference between neighboring sites is much less than $k T$, so it is natural to ask how weak fields affect domain growth. In the zero field limit, our model corresponds to standard Lifshitz-Slyozov growth with asymptotic $L \sim t^{1 / 3}$ behavior. Slightly away from this limit, we find that low fields produce such slow coarsening for a while, and then generate 
a crossover to fast growth and noticeable anisotropy. Figure 11 shows this behavior. First, initial transients die away on a time scale given by the rate of motion for $q=3$ atoms (as in [5]), and $t^{1 / 3}$ growth sets in. When the characteristic domain size is still much less than $k T / 2 \Delta$, growth takes off and the presence of the field also appears in a loss of square domain symmetry. Figure 12 shows rough visual estimates of crossover length as a function of field. Although this plot may include systematic error from pinpointing a crossover in increasingly rounded curves, it strongly suggests that the crossover length has a weak dependence on field.

To gain a physical understanding of the crossover, first note that it represents a transition between diffusion-dominated growth, and driven collisions produced by the external field. In this low field regime, where the potential drop across the domain is still less than $k T$, we can describe the driven motion with linear response theory. We will argue that crossover occurs when a typical block absorbs more vacancies through concerted motion along the field than through diffusive motion. Driven collisions should win when the area of a circle swept out through diffusion, $\pi D t$, is equal to the area swept out by linear motion, i.e.

$$
\pi D t=v \cdot t \cdot w
$$

Note that we can replace the horizontal length scale $w$ with a general length scale $L$, since this early growth regime is precisely when length scales in all directions are the same.

To describe the crossover more completely, we need to know how $D$ and $v$ vary with the size of a domain in our model. For velocity, we refer back to the section describing exponential growth, where we found $v \sim L$ whenever motion was limited by the slow rate of dislodging the first atom from a row. To describe the variation of the diffusion constant, note that $D \cong \omega(\Delta x)^{2}$, where omega is the frequency of a typical move and $\Delta x$ is the center of mass displacement caused by such a move. For a faceted domain, a typical move takes an atom from one rare kink in the boundary to another. Such a move displaces the atom by a distance of order $L$ and the center of mass by $\Delta x \sim 1 / L$. Since the frequency of these moves will be proportional to $L$, we expect that faceted domains will have $D \sim 1 / L$. Note 
that these results for $v$ and $D$ are consistent with the Einstein relation that should apply at such small fields: $D / 2 k T=v /$ force. (The driving force will be proportional to total charge of a block and therefore its area.)

Plugging these results into equation (6), we find that the prediction $v \cdot L_{\text {cross }}=\pi D$ becomes $\Delta \cdot L_{\text {cross }}^{2} \propto L_{\text {cross }}^{-1}$ or $L_{\text {cross }} \propto \Delta^{-1 / 3}$. [20] If this relationship correctly describes the crossover to fast growth, then simulations with a well-developed $L \propto t^{1 / 3}$ growth before crossover should follow the scaling collapse $L(\Delta, t)=\Delta^{-1 / 3} \mathcal{L}(t \Delta)$, at least until fast growth has taken over. Figure 13 shows such a collapse. Considering the numerical difficulty of achieving well-developed $t^{1 / 3}$ growth for a wide range of fields, we believe an $\Delta^{-1 / 3}$ crossover is supported by the data; in any case, our results strongly indicate that length scale at crossover varies only weakly with field.

\section{CONCLUSIONS}

Thus, in our model, a reduction in external field only produces a small increase in the

minimum domain size for takeoff. Although the departure from $t^{1 / 3}$ growth appears to be small at low fillings, the field-driven takeoff at higher fillings soon leads to large empty regions which move steadily against the field as particles sweep quickly through their centers. For moderately high lattice fillings, fast growth involves most of the vacancy regions. Here, domain growth is very roughly linear in time, and horizontal correlations come close to scaling. At very high lattice fillings and low temperatures, fewer domains undergo significant coarsening, but those that do have runaway, exponential growth. In our model, explosive growth ends when domains are large enough to have rough bases, either through thermal effects or constant collisions. Our study has also explored the strong impact which faceting can have on size dependence in velocities of vacancy clusters. This is a subject with potential applications in the study of void electromigration in small aluminum interconnects, where faceting is well documented and voids have been observed to move through the middle of single crystal grains [21]. Most importantly, our model demonstrates that external driving 
forces can be surprisingly effective in producing domain clumping and macroscopic particle fluxes.

How such enhancement plays out in particular experimental systems is still an open question. In the instance of Moeckly's YBCO electromigration experiments, particle motion takes place in the anisotropic environment of the oxygen "chain" planes, and our simple model does not incorporate such inherent anisotropies. Also, associating our model with the YBCO experiment involves abstracting our simulated phase separation of completely filled and empty areas to an experimental phase separation which may be less extreme. Neutron and TEM observations of YBCO suggest that domain segregation in these planes produces regions of more closely spaced oxygen rows and less closely spaced rows [22]. Still, the more open environment of widely spaced rows does allow increased mobility [23]. Moeckly's observations of large oxygen-depleted regions suggest that field-induced clumping is vital component of his experiments. Without describing the specific characteristics of YBCO, our model provides a qualitative check that small external driving forces can indeed facilitate the segregation of domains with high particle mobility.

This study serves as a preliminary survey of a broad range of interesting and potentially relevant model behaviors. An extension to three dimensions would allow us to study the effect of a finite-temperature roughening transition. Our study of faceting effects should be expanded to cover other types of dynamics, such as those which facilitate surface diffusion. Our simple picture of coarsening in the presence of scaling clearly needs to be modified to include departures from scaling and fluctuations in domain velocity. And an entire regime of low filling needs to be explored and understood. Our work illustrates the breadth of issues involved in studying how a separation of time-scales due to faceting can affect response to an external driving force. We have demonstrated that useful approaches to this problem may be found outside the long wavelength, late time limit. Further work should improve our understanding of particle motion and domain coarsening in systems which, instead of being conveniently isolated in a thermal bath, are knocked out of equilibrium by an external force. 
We would like to thank B. Moeckly, R. Buhrman, J. Marko, and G. Barkema for helpful conversations. This work was partly funded by the NPSC (LKW), and the NSF under grant DMR-91-18065 (LKW, JPS). This research was conducted using the resources of the Cornell Theory Center, which receives major funding from the National Science Foundation (NSF) and New York State. Additional funding comes from the Advanced Research Projects Agency (ARPA), the National Institutes of Health (NIH), IBM Corporation, and other members of the center's Corporate Research Institute. (Roughly 1000 IBM SP1 processor hours were used.) 


\section{REFERENCES}

[1] J. D. Gunton, M. San Miguel, and P. S. Sahni, in Phase Transitions and Critical Phenomena, Vol. 8, eds. C. Domb and J. L. Lebowitz (Academic, New York, 1983) p. 267. For a more recent review, see A. J. Bray, Adv. Phys., to be published.

[2] K. Binder and D. Stauffer, Phys. Rev. Lett. 33, 1006 (1974).

[3] J. L. Lebowitz, J. Marro, and M. H. Kalos, Acta Metall. 30, 297 (1982).

[4] I. M. Lifshitz and V. V. Slyozov, J. Phys. Chem. Solids 19, 35 (1961).

[5] G. T. Barkema, J. F. Marko, and J. de Boer, Europhys. Let., 26, 653, (1994).

[6] S. Katz, J. L. Lebowitz, and H. Spohn, Phys. Rev. B 28, 1655 (1983); J. Stat. Phys. 34, 497 (1984).

[7] B. Schmittmann, Int. Jour. Mod. Phys. B 4, 2269 (1990).

[8] K. -T. Leung, J. Stat. Phys. 61, 1744 (1988).

[9] D. H. Boal, R. P. K. Zia, and B. Schmittmann, Phys. Rev. A 435214 (1991); C. Yeung, T. Rogers, A. Hernandez-Machado, and D. Jasnow, J. Stat. Phys. 66, 1071 (1992).

[10] C. Yeung, T. Rogers, A. Hernandez-Machado, and David Jasnow, J. Stat. Phys. 66, 1071 (1992).

[11] K. Kawasaki, Phys. Rev., 148, 375 (1966).

[12] S. Ohta, A. Natori, and H. Yasunaga, Surf. Sci., 265, 252 (1992).

[13] B. H. Moeckly, D. K. Lathrop, and R. A. Buhrman, Phys. Rev. B 47, 400 (1993); B. H. Moeckly, P. E. Sulewski, and R. A. Buhrman, Appl. Phys. Let., 64, 1427 (1994).

[14] G. Cannelli, R. Cantelli, F. Cordero, and F. Trequattrini, Supercond. Sci. Technol. 5, $247(1992)$.

[15] S J. Rothman, J. L. Routbort, and U. Welp, Phys. Rev. B, 44, 2326 (1991). 
[16] J. R. LaGraff and D. A. Payne, Physica C, 212, 470 (1993).

[17] Note that large $\Delta$ means that more Monte Carlo moves are thrown away. Thus, to get a field-independent measure of time, we must scale the Monte Carlo time by $e^{-2 \Delta}$.

[18] Two processes tend to make domains fatter. They can combine sideways with other domains, and the column of atoms at the side of a domain can come loose and be swept up to the top of the domain by the field. Widening continues until these processes are balanced by the rate at which pile-ups of atoms at the top corners of a domain can grow to refill a side column of particles.

[19] Note that this procedure only gives a precise measure of length scale in a scaling regime, where the basic shape of the correlation function remains constant. In the present regime, the outer tail of the correlation curve grew slightly more quickly than the midsection.

[20] Note that if domains are rough and $v$ is independent of $L$, we expect $D \propto L^{-2}$. This still gives $L_{\text {cross }} \propto \Delta^{-1 / 3}$. See Gunton's article in [1] for a discussion of various diffusion mechanisms for rough domains.

[21] S. Shingubara and Y. Nakasaki, Appl. Phys. Lett, 58, 42 (1991). J. H. Rose, Appl. Phys. Lett, 61, 2170 (1992).

[22] R. J. Cava, et al., Physica C, 165, 419 (1990). S. Amelinckx, G. Van Tendeloo, and J. Van Landuyt, Solid State Ionics, 39, 37 (1990). J. Reyes-Gasga, T. Krekels, G. Van Tendeloo, et al., Physica C, 159, 831 (1989).

[23] J. R. LaGraff and D. A. Payne, Physica C, 212, 487 (1993). 


\section{FIGURES}

FIG. 1. Above are snapshots from our simulation. An external field pushes white particles up. Pictures (a) and (b) are early and late configurations from a run with $90 \%$ filling. Here, a few of the black vacancy regions undergo runaway growth as they sweep down through the lattice. Pictures (c) and (d) show early and late stages of a run with $70 \%$ filling. Here, blocks of all sizes are moving, and domain area growth is close to linear in time. All pictures have $\Delta=1$ and $J=1.5$. Animations of our simulations are available at http://www.lassp.cornell.edu/ LASSP_Science.html (under "Coarsening in a Driving Force").

FIG. 2. From bottom to top, these plots give vertical and horizontal cluster size, and the product of the two as a function of time. (Sizes are taken as the FWQM of two-point correlation functions, such as those in Figure 5.) These simulation results correspond to $90 \%$ filling and $\Delta=1$. They were produced with a fast, low temperature algorithm. Time is scaled so that a given triply coordinated atom will wait approximately one time unit before moving. Note that the plot has log of cluster size vs linear time, so that a straight line indicates exponential growth. Growth slows at late times, when domains become so large that they have rough bases.

FIG. 3. From simulations with a single domain in an empty lattice: velocity as a function of domain width for (a) $J=2$, and (b) $J=1$. Note that the low temperature (high $J$ ) results are nearly linear. Data are averages of 30 runs; error bars represent estimated variations in the mean. $\Delta=$ 1.

FIG. 4. Cluster width vs time in a $90 \%$ full lattice at three temperatures. These plots were produced with standard finite-temperature dynamics, and time is again scaled so all $q=3$ atoms have moved approximately once at $t=1$. The circles have $J=1.5$ and show exponential growth which crosses over to power law at late times. The diamond plot (lowest) has $J=1$ and does not show well formed exponential growth. The squares have $J=1.25$ and show an intermediate behavior. $\Delta=1$. 
FIG. 5. Log-linear plot of cluster area vs time. From top to bottom the curves correspond to $80 \%, 82 \%$, and $85 \%$ filling. $\Delta=1$.

FIG. 6. Best collapse of horizontal(a, c) and vertical(b, d) correlation functions. The first set of collapses is for $70 \%$ filling and covers a factor of 9 in time. The second set is for $60 \%$ and covers a factor of 7.5 in time. $\Delta=1$ and $J=1.5$. Note that the vertical correlation function shows that the regions sandwiched between vacancy domains are becoming somewhat more swept out. The later curves are clustered together at the bottom of the collapse.

FIG. 7. From left to right, these curves correspond to $60 \%, 70 \%$, and $80 \%$ filling. In these log-log plots of cluster area vs time, the asymptotic slopes vary from about 1.3 at $60 \%$ filling to about 1.5 at $70 \%$ and at $80 \%$ filling, corresponding to $\zeta=.65$ and $\zeta=.75 .(J=1.5$ and $\Delta=1$. $)$

FIG. 8. From simulations with a single domain in an empty lattice: velocity plotted against inverse domain width. These results are consistent with velocities which approach $v=v_{0}-v_{1} / w$ at large domain size, as shown by the linear fit. Here, $J=1$ and $\Delta=1$.

FIG. 9. Velocity as a function of inverse width in an actual simulation run with $J=1.5$ and $\Delta=1$. Despite poor statistics, the linear fit is consistent with a $1 / w$ correction to a terminal velocity, as in Figure 8.

FIG. 10. Preliminary results for power law in cluster area vs time at 20\%, 30\%, 50\%, 60\%, and $70 \%$ filling. At each time, an approximate logarithmic derivative in cluster area vs time is shown. (This is an effective slope over a finite time window around each given time in a curve such as those in figure 7.) Note that the asymptotic power increases with filling.

FIG. 11. Crossover to fast growth for $90 \%$ filling and $\Delta=.1$. The circles show characteristic domain width and the squares show domain height. The straight line shows $L \sim t^{1 / 3}$ growth.

FIG. 12. Rough visual estimates of crossover length versus external field for several fields at $80 \%$ filling. The straight line shows $L \propto \Delta^{-1 / 3}$ for comparison. 
FIG. 13. Collapse of cluster width vs time for several low field curves, ranging from $\Delta=.005$ to $\Delta=.1$. The collapse follows $L(\Delta, t)=\Delta^{-1 / 3} \mathcal{L}(t \Delta)$, and uses runs with $80 \%$ filling. 


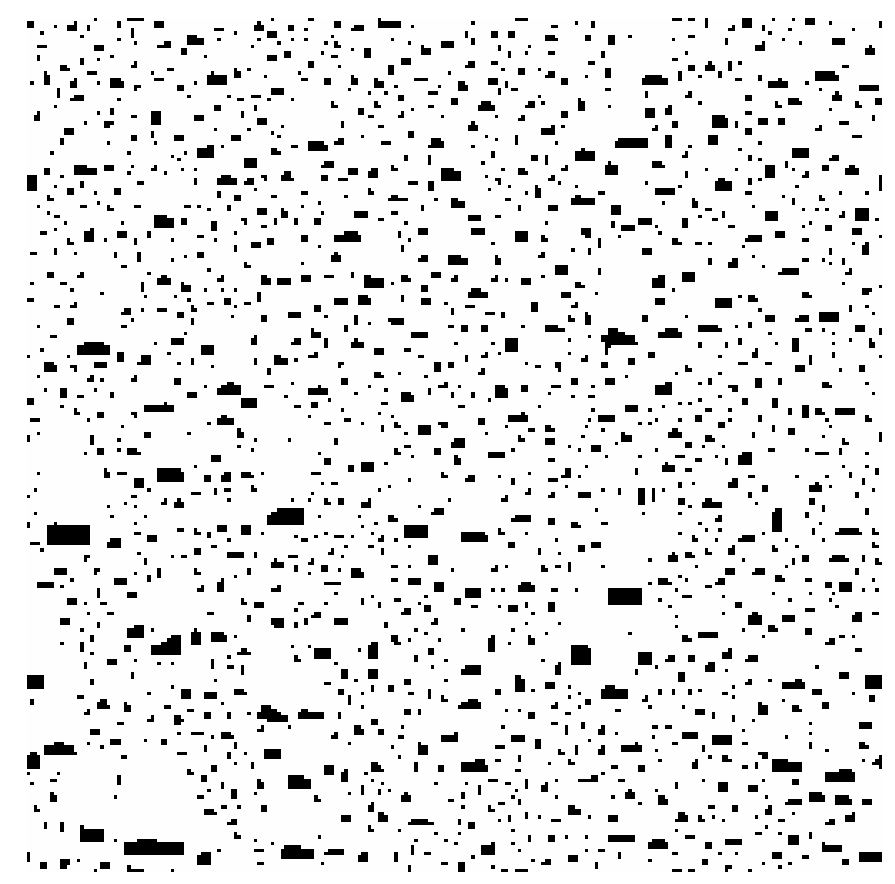




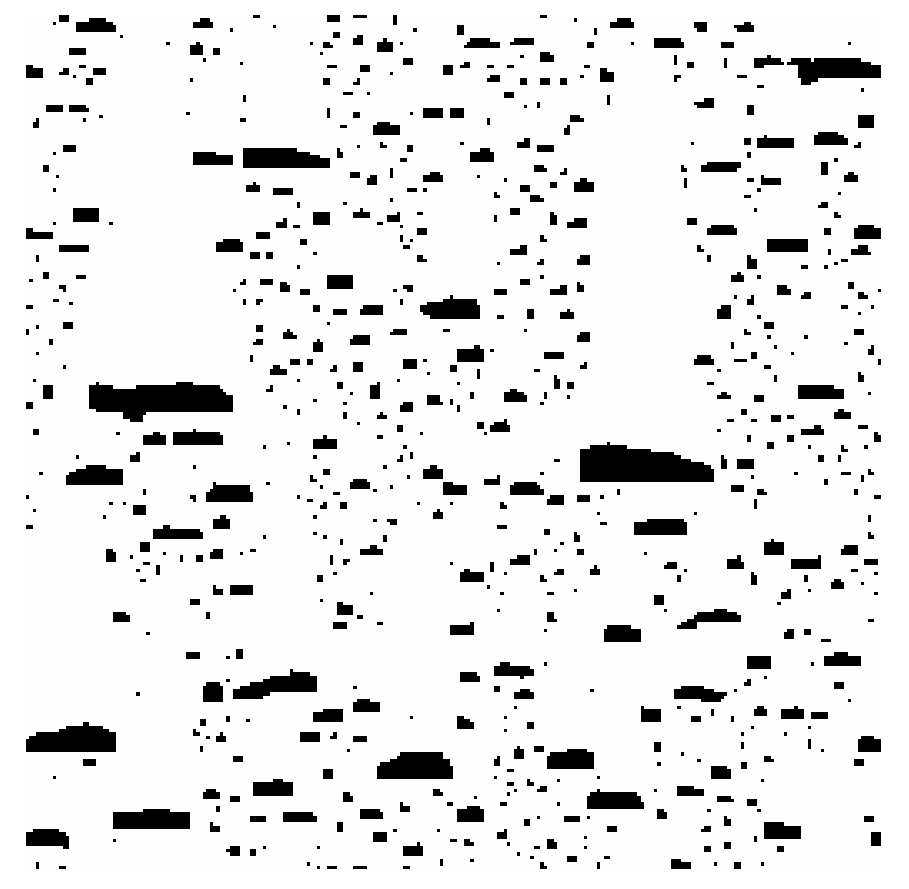




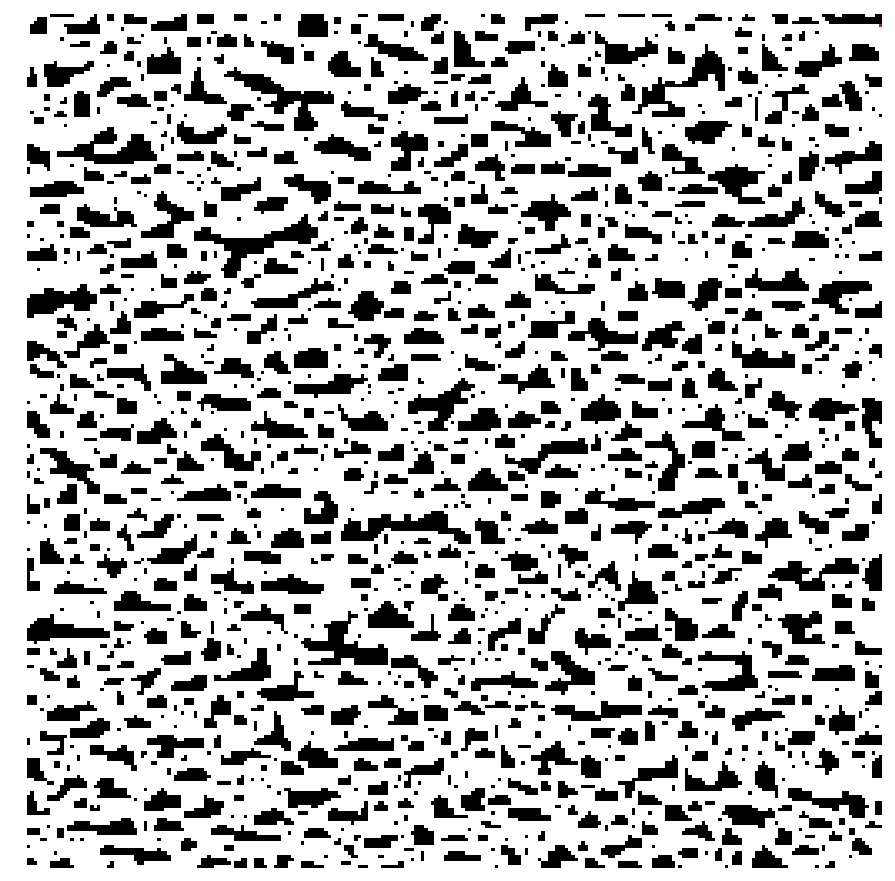




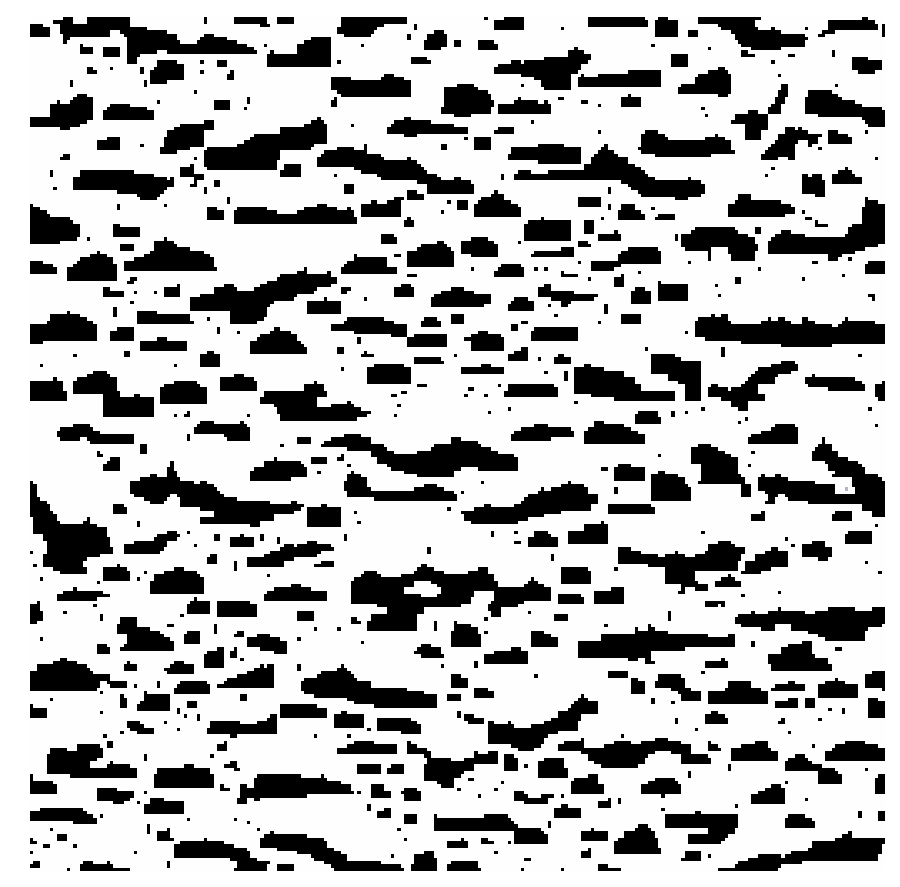




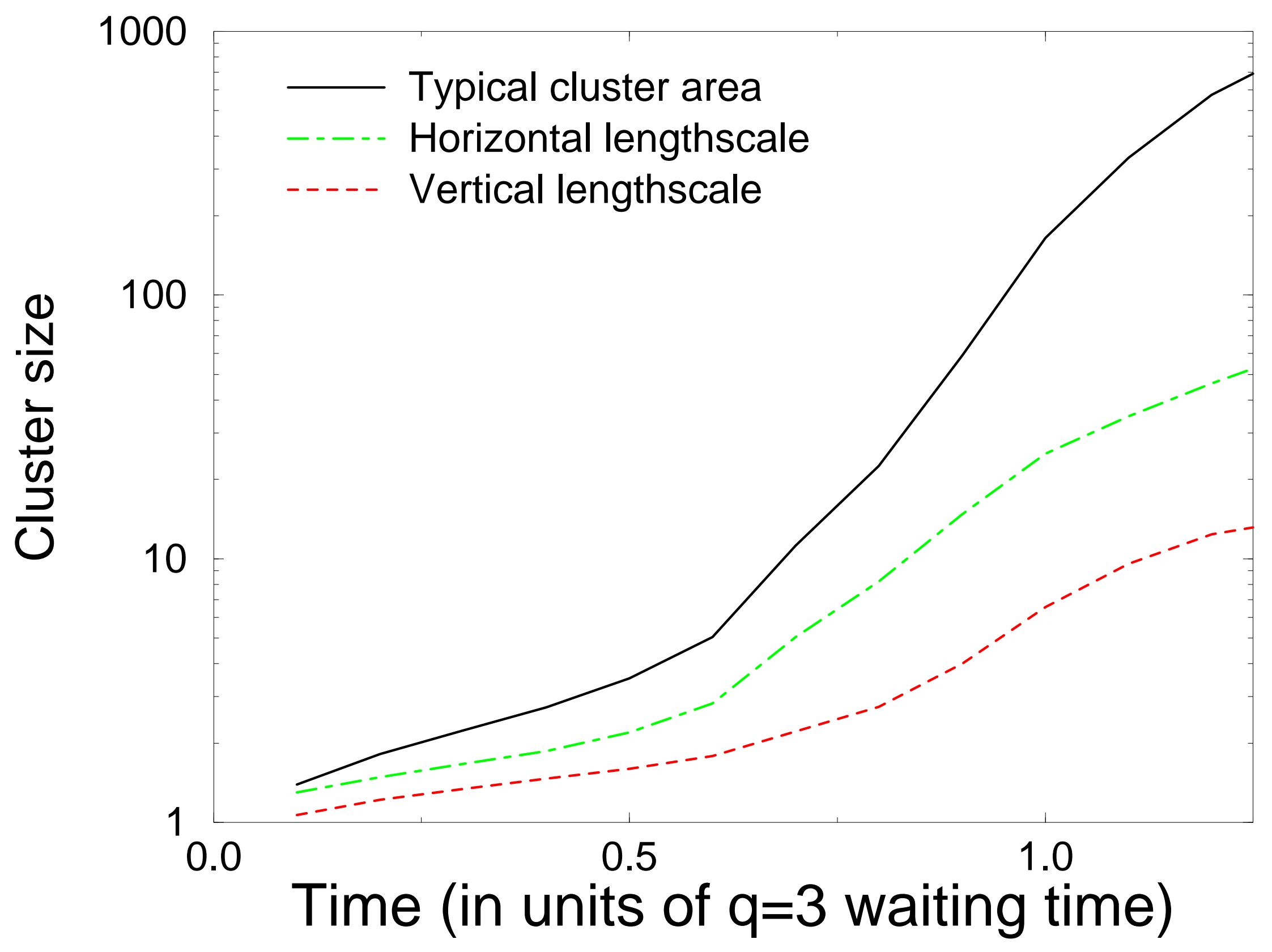




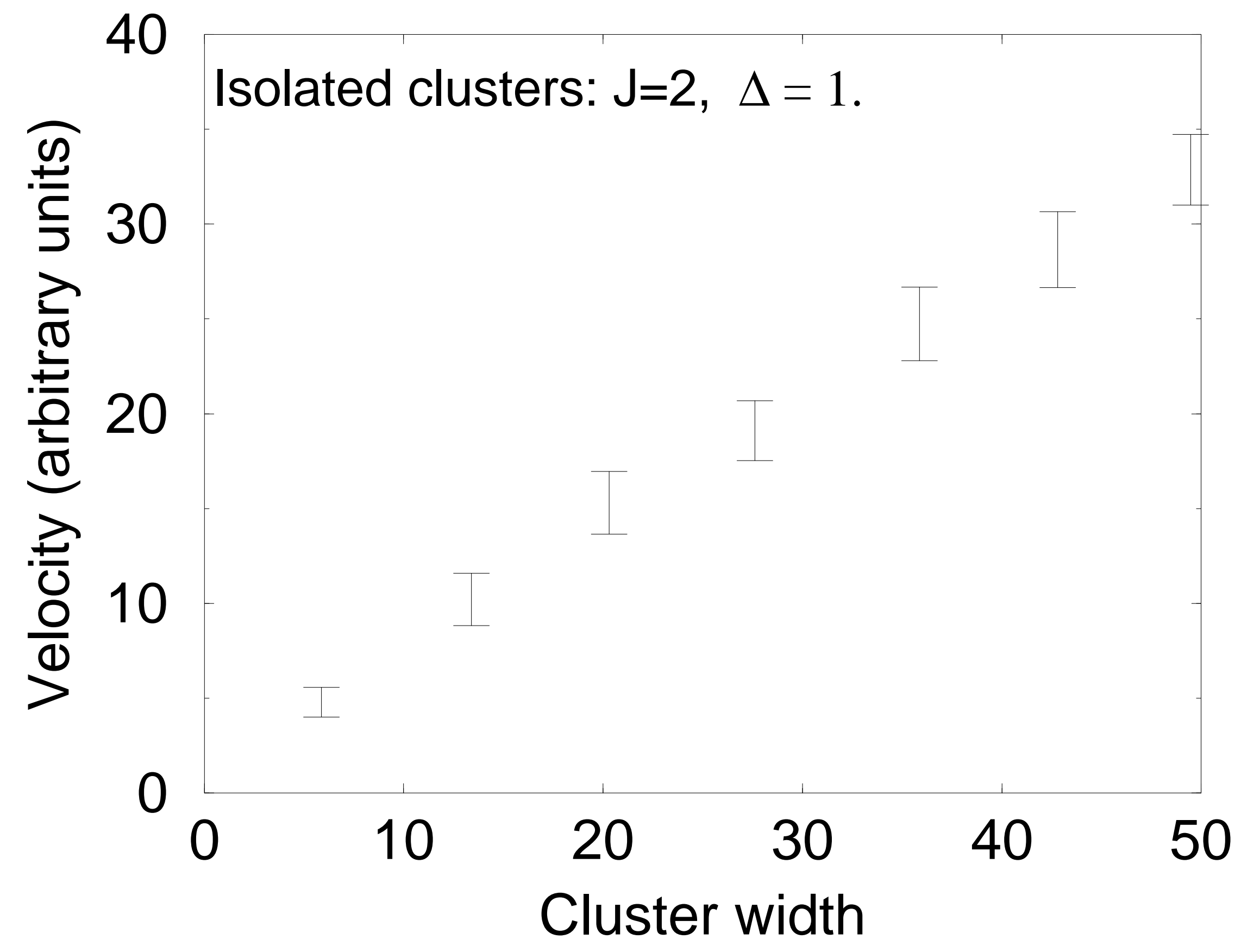




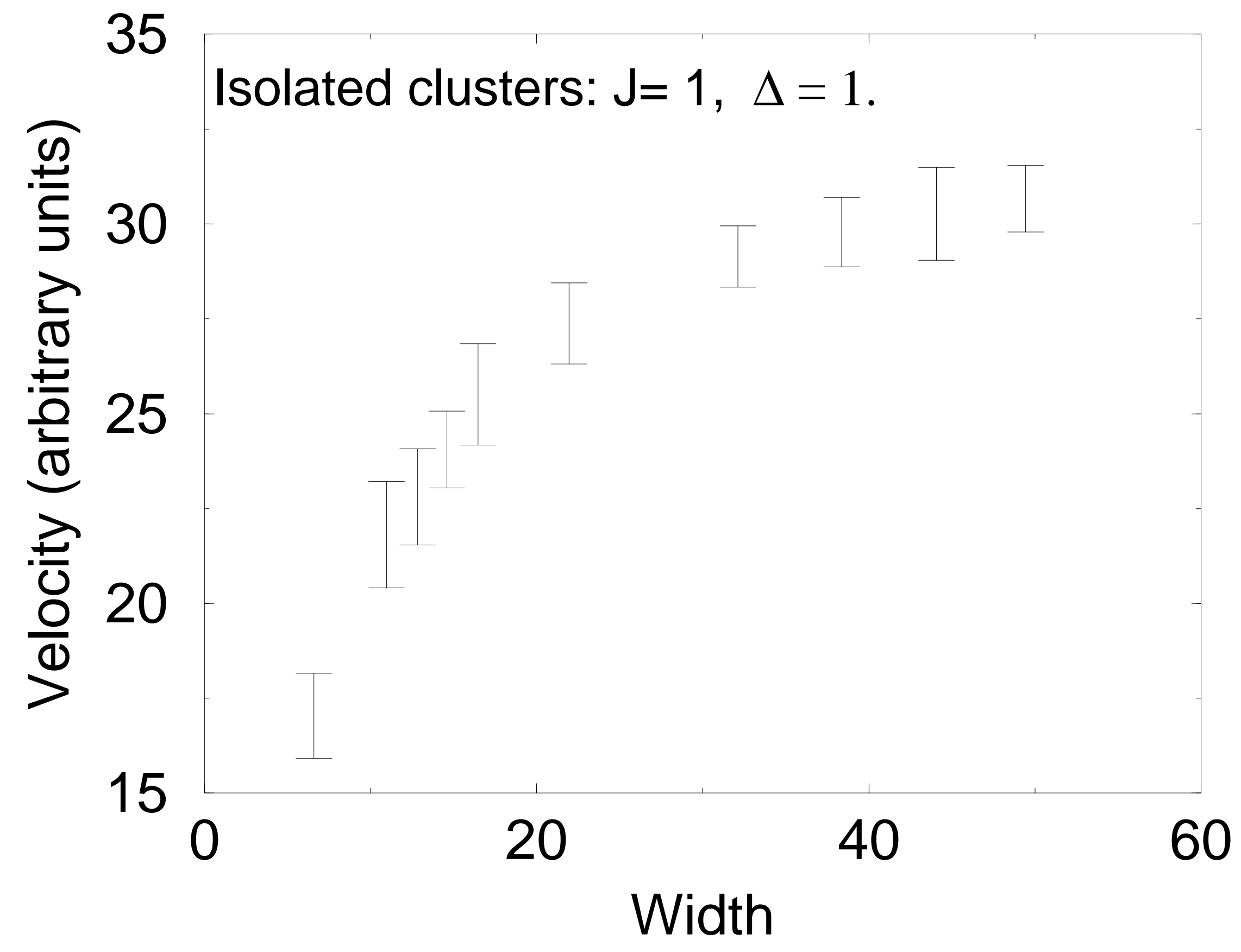




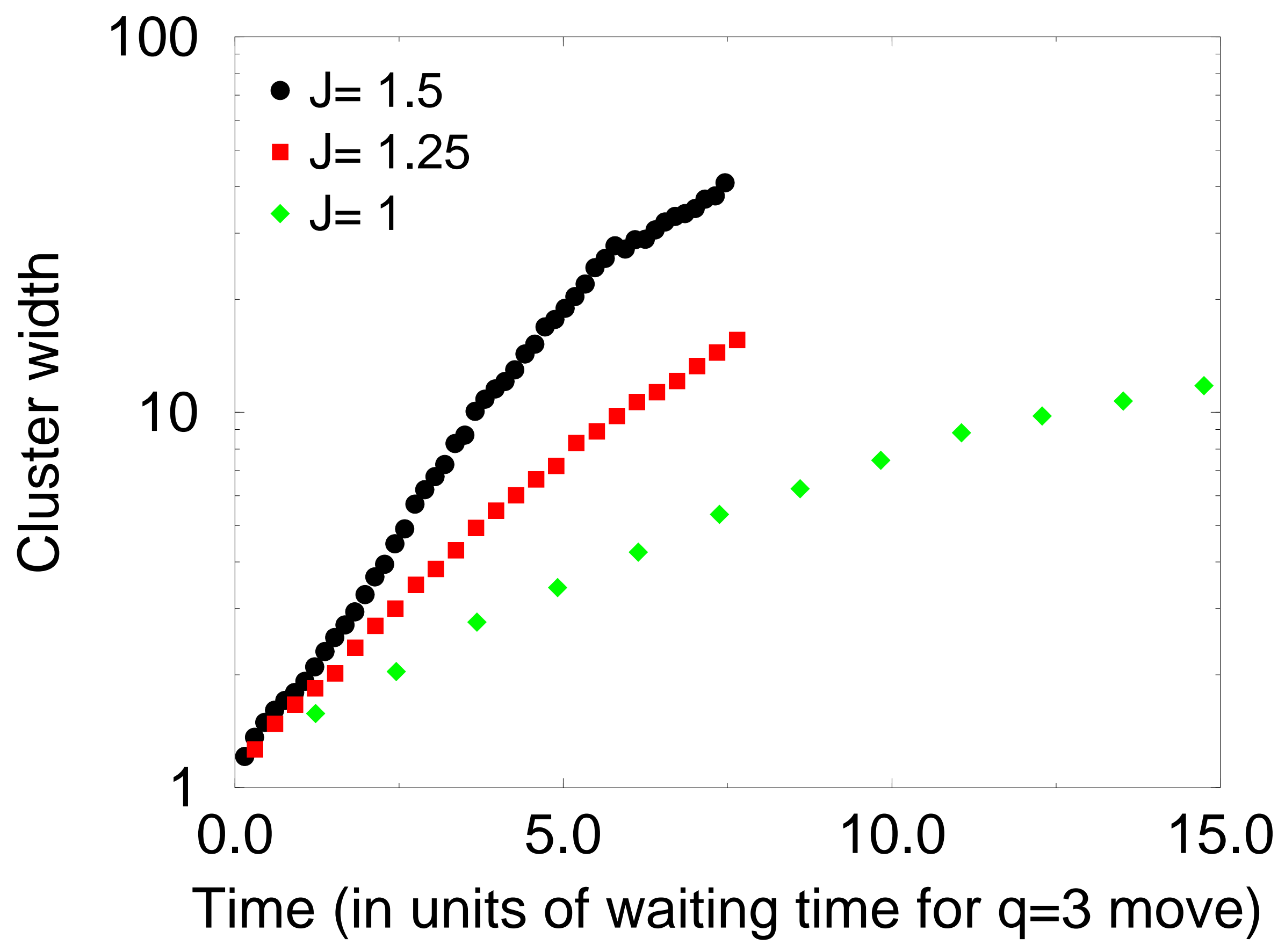




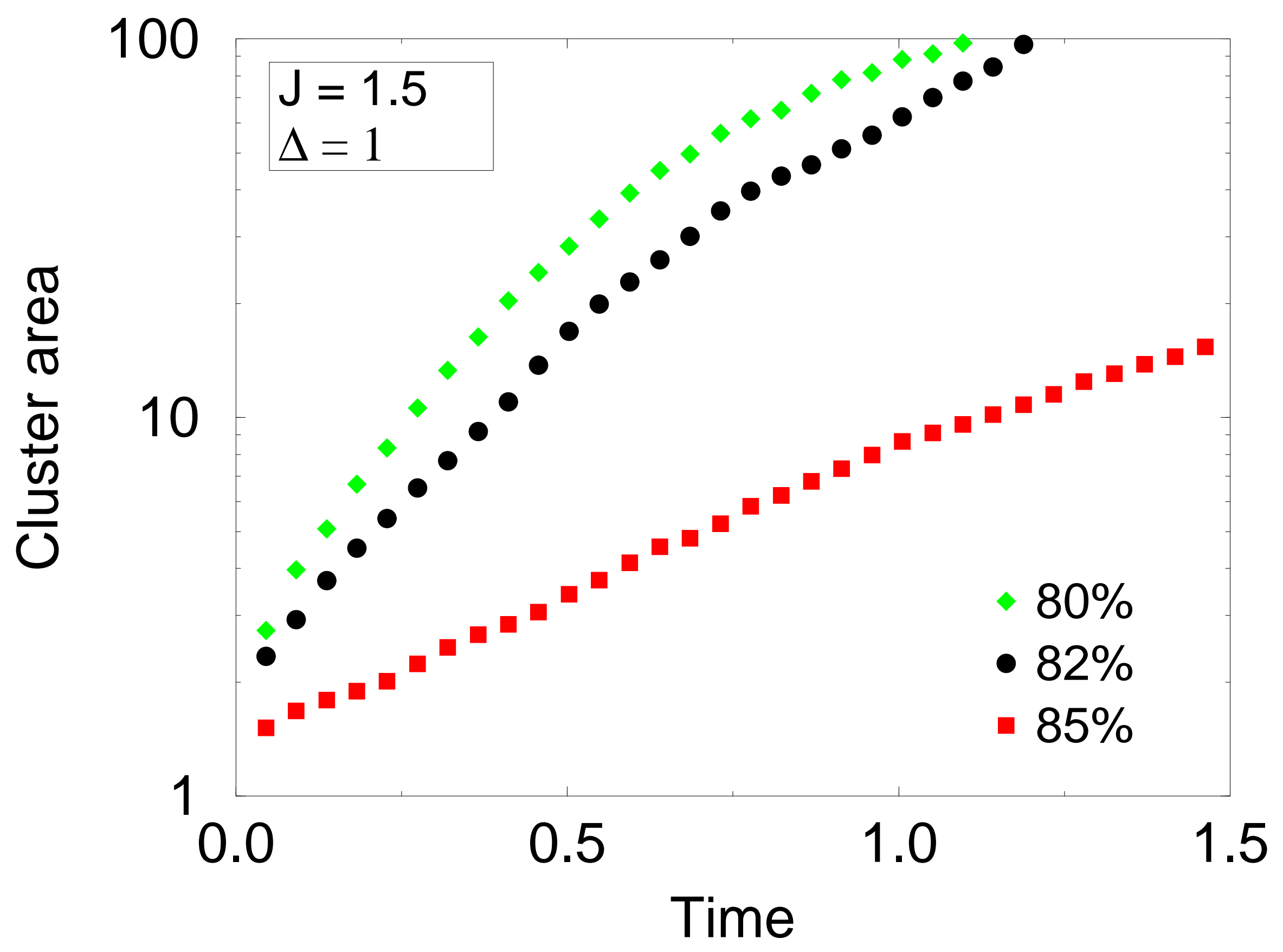




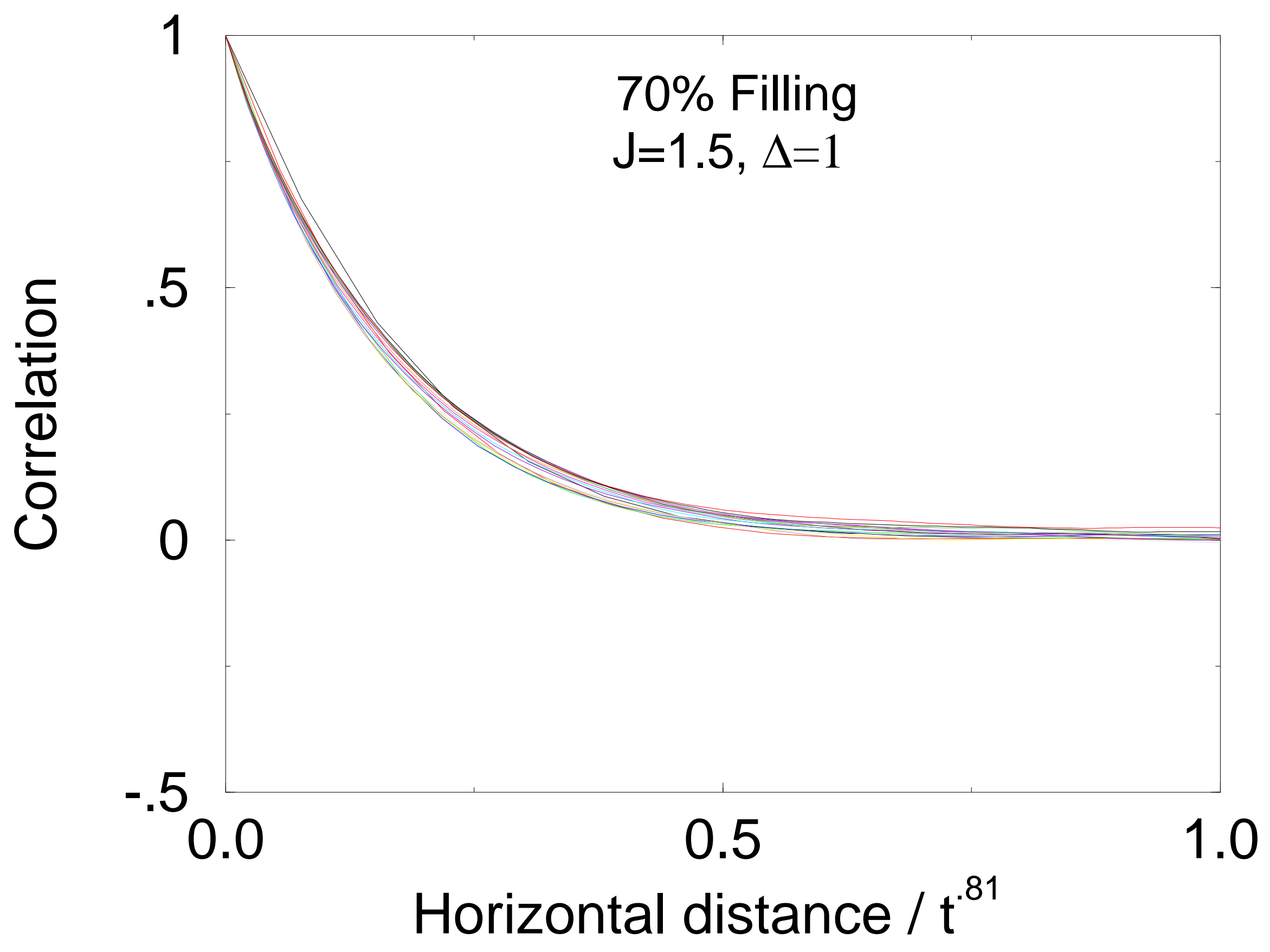




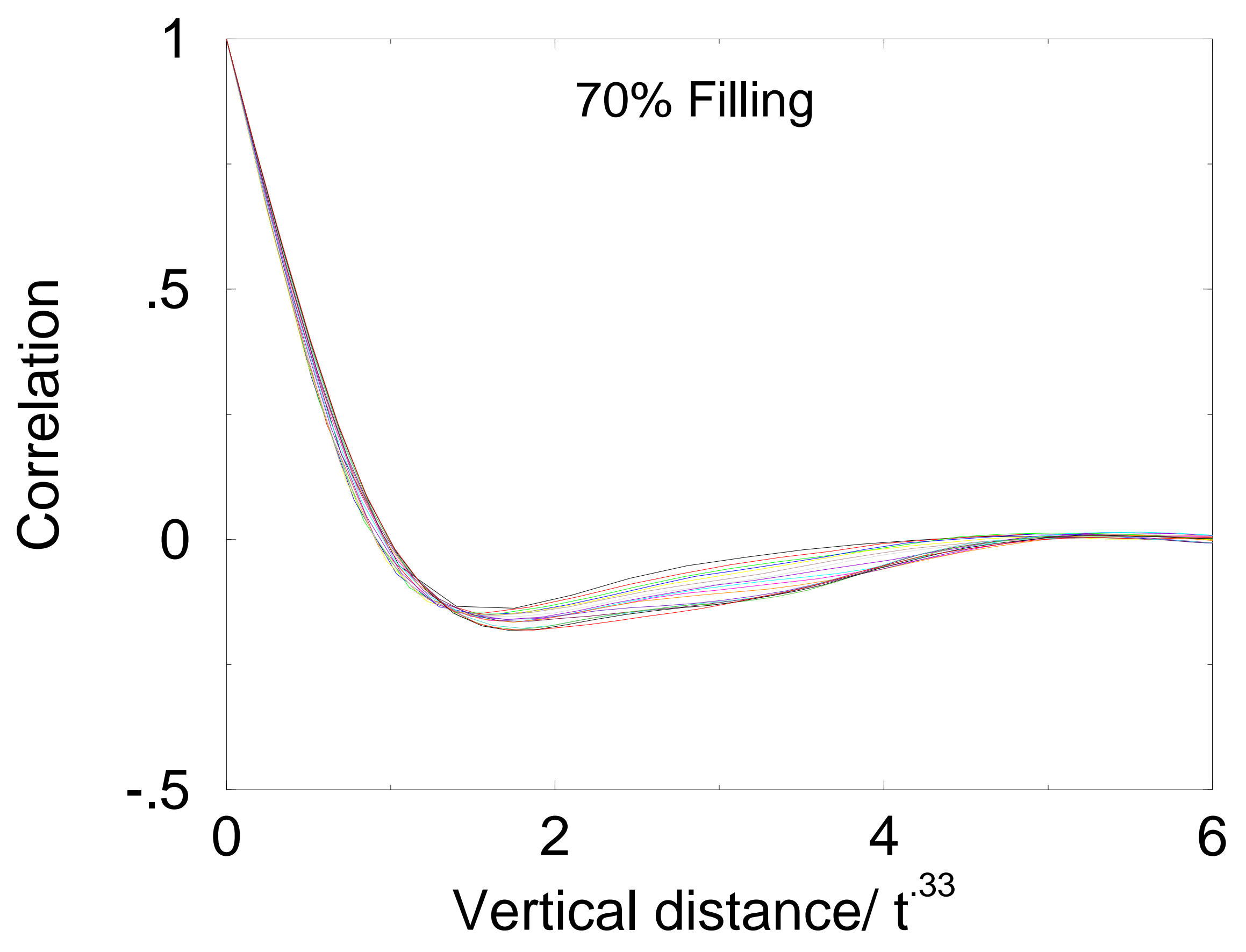




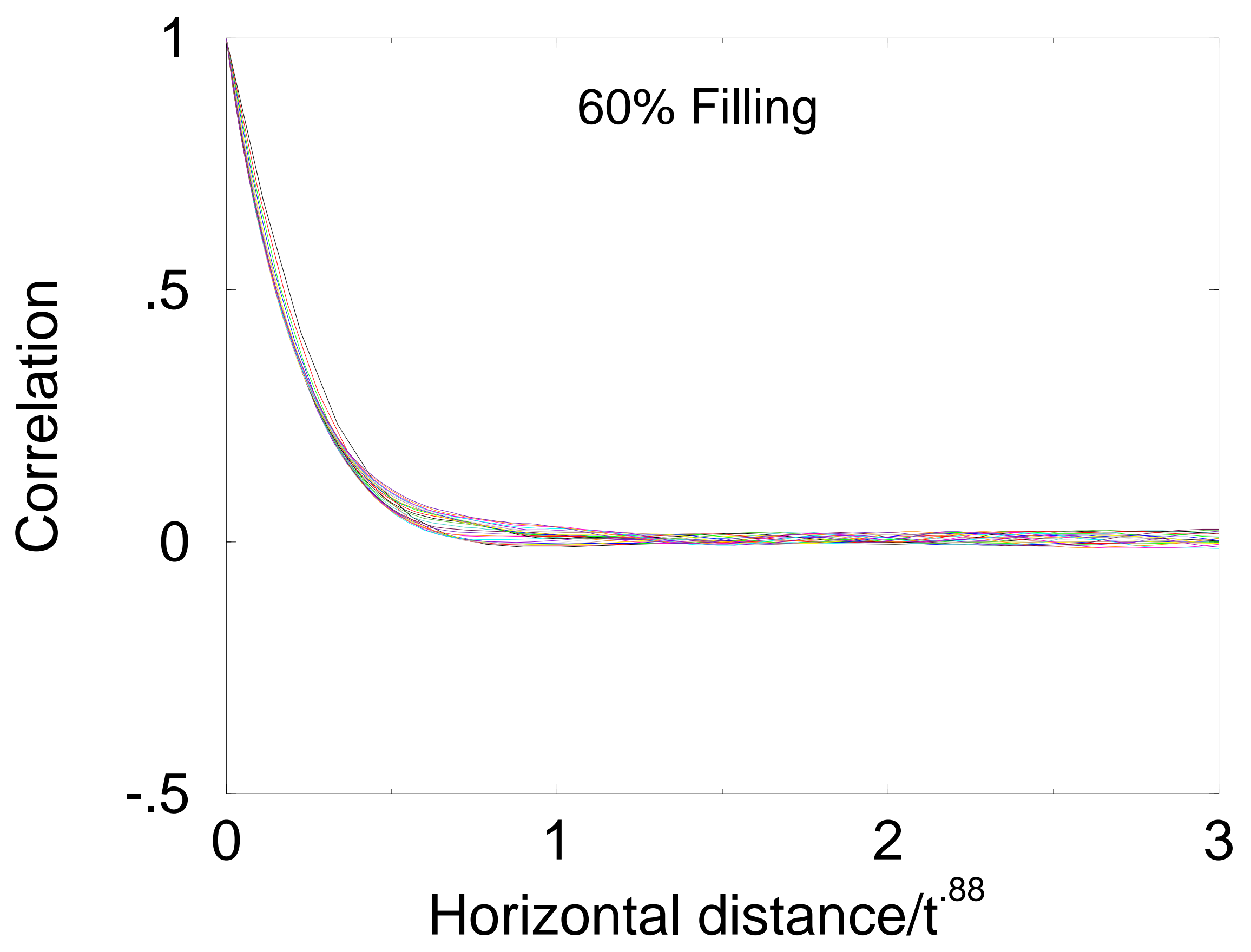




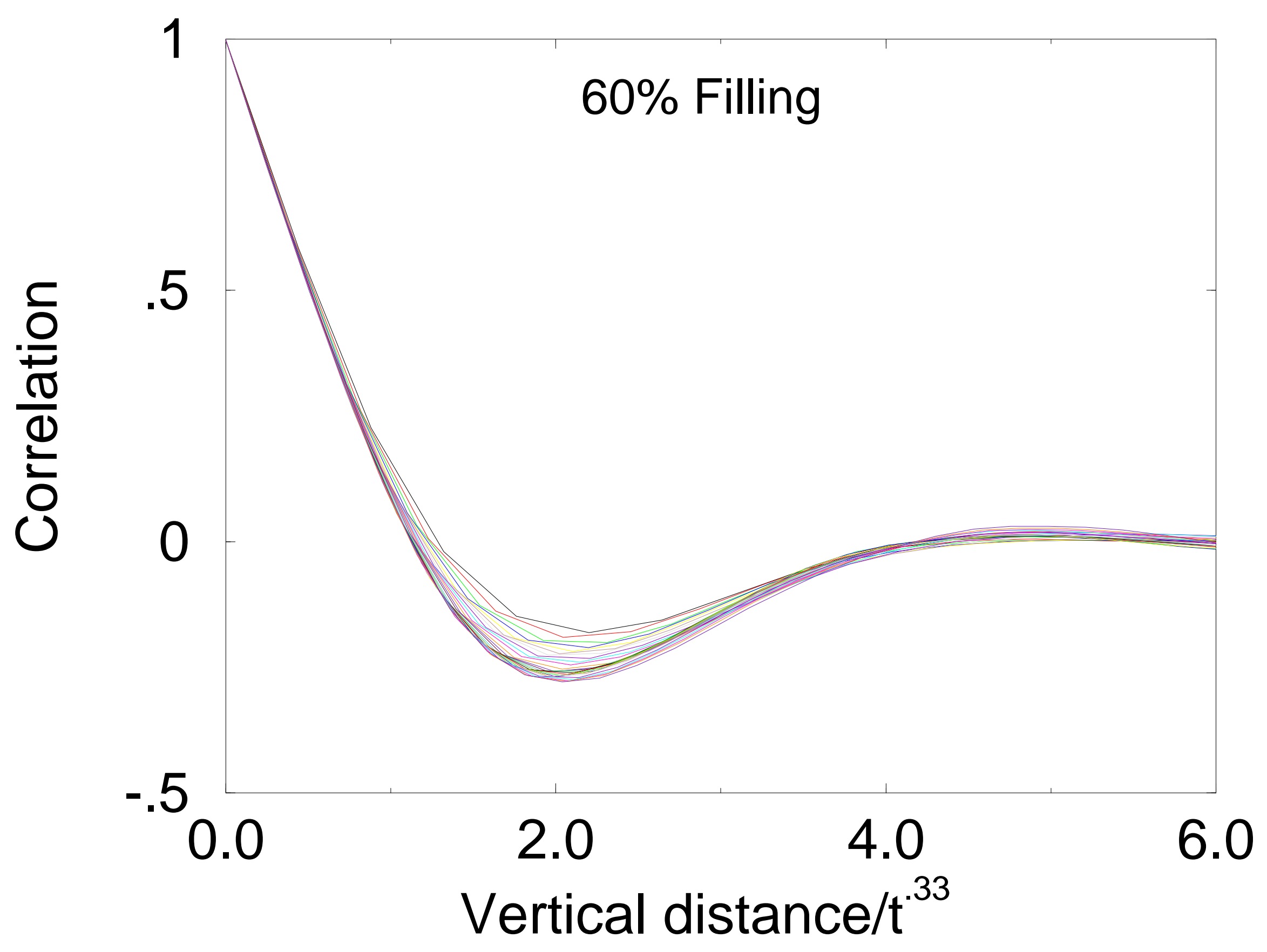




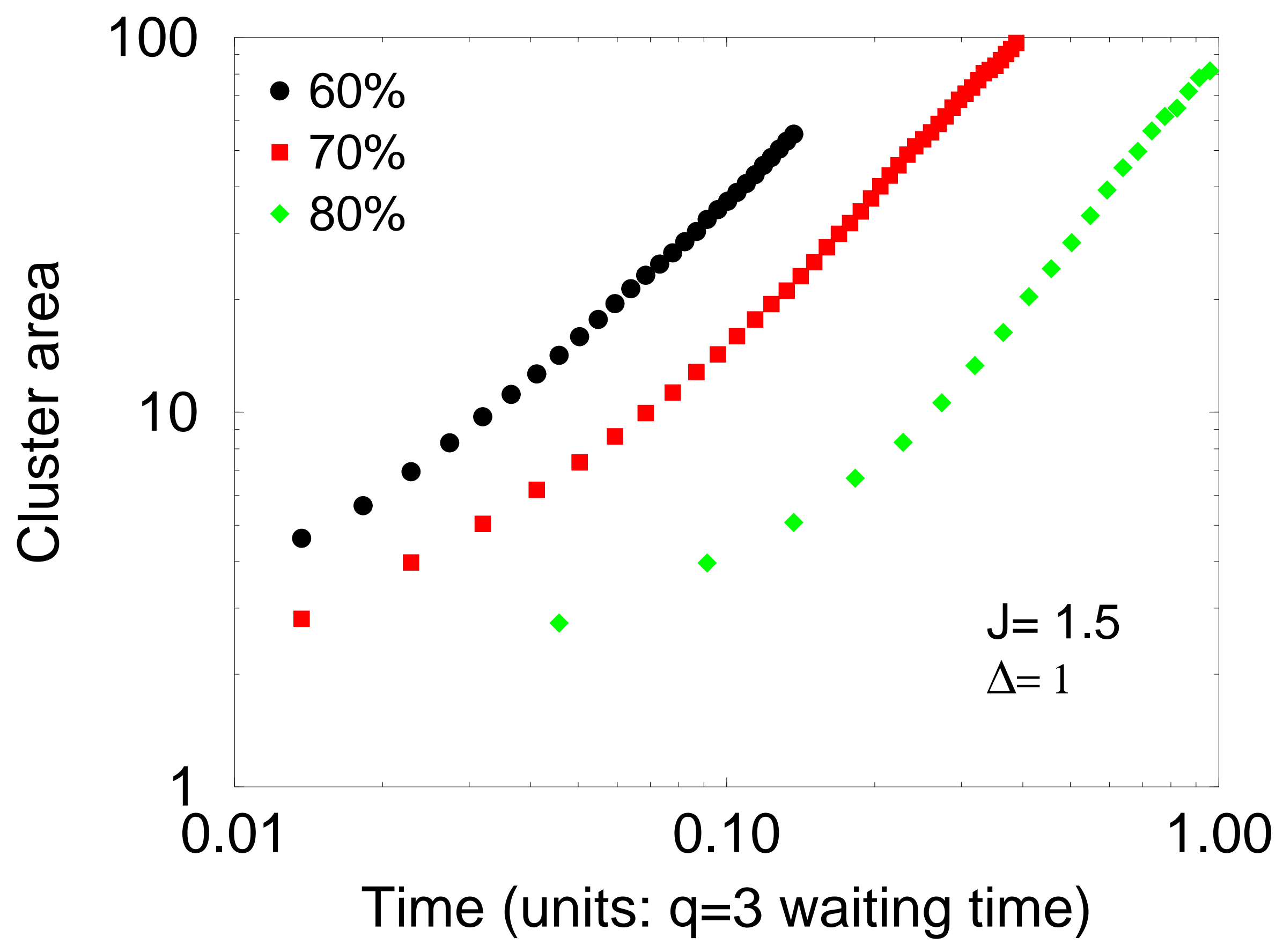




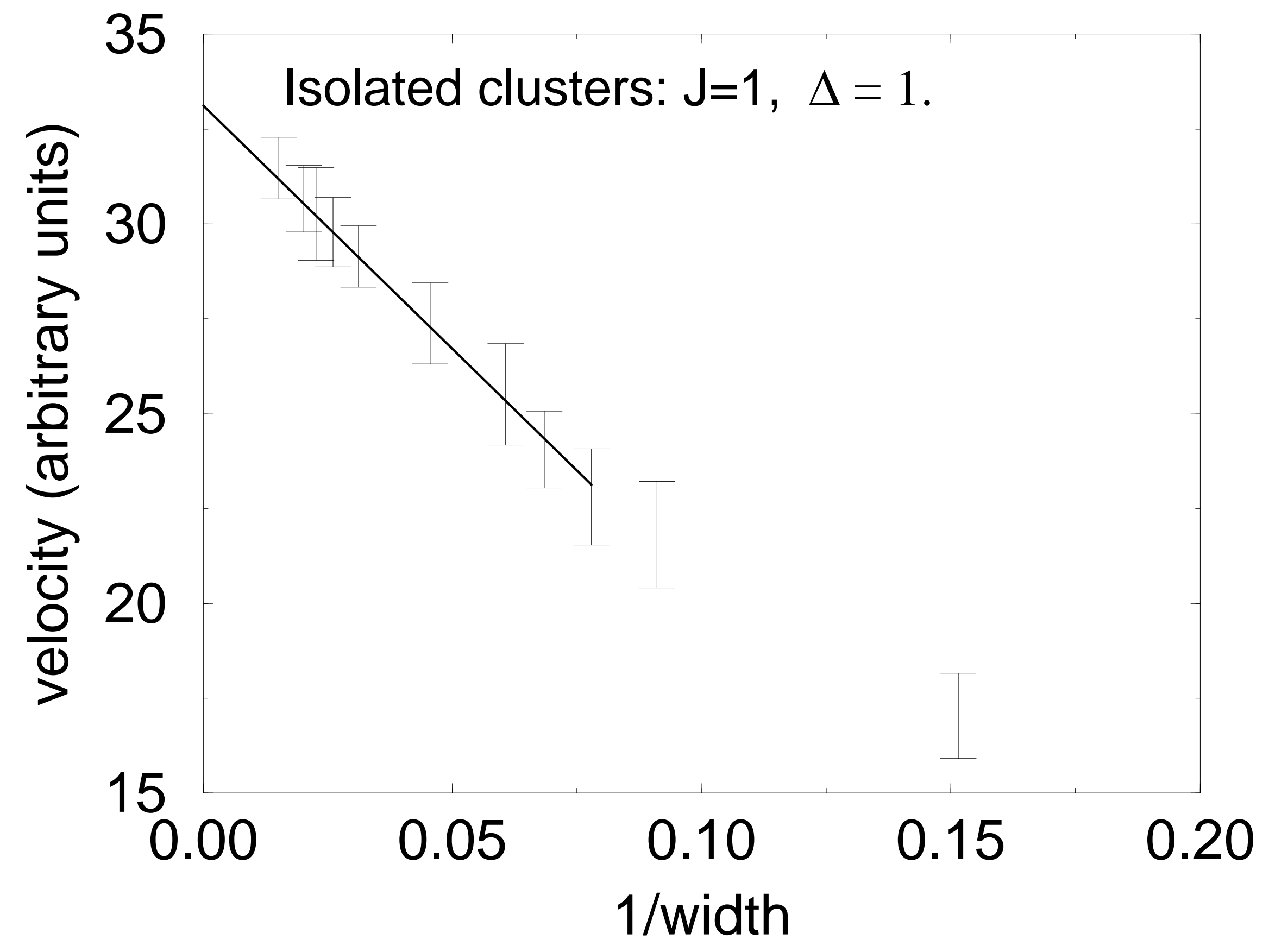




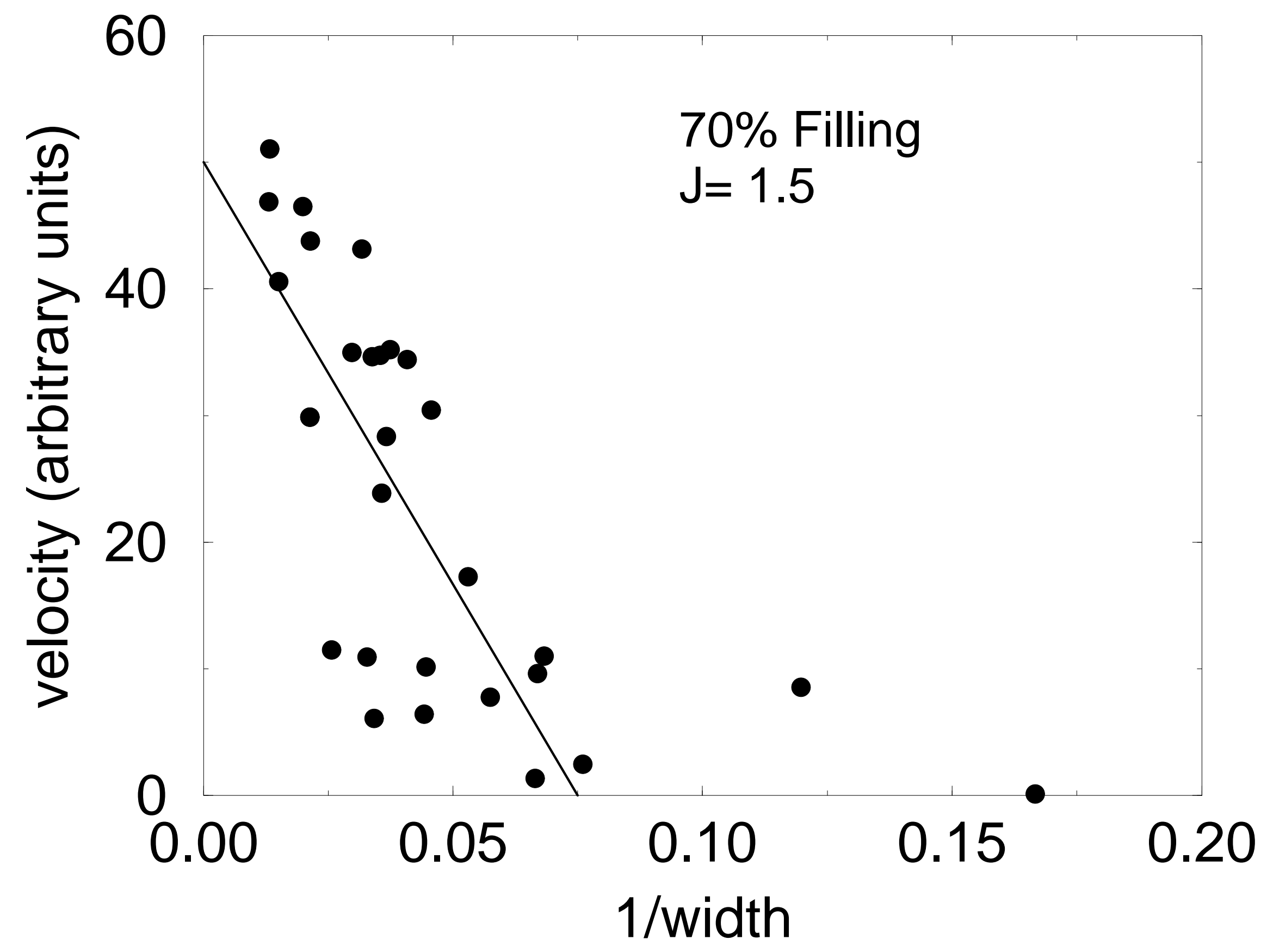




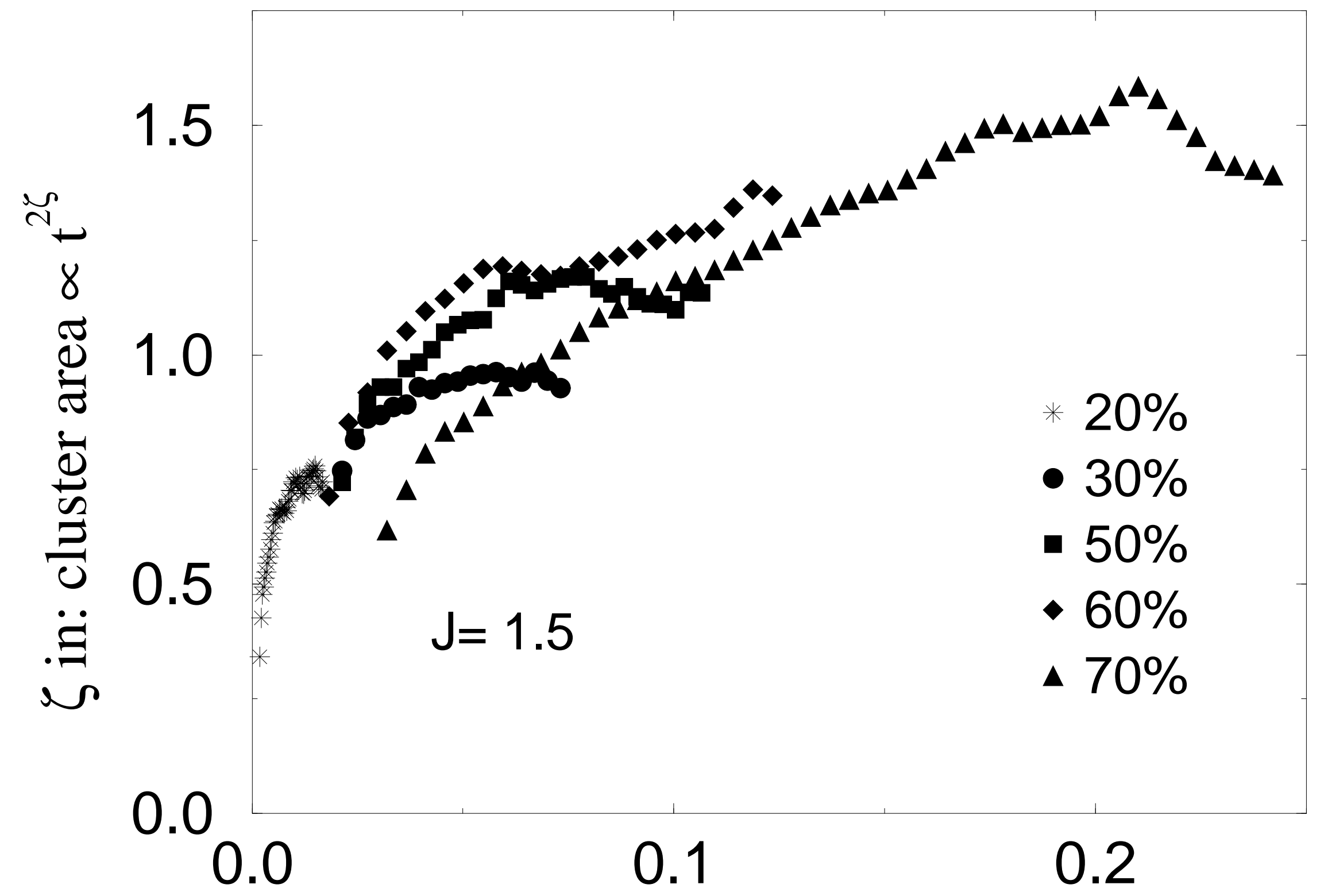

Time (units: $q=3$ waiting time) 


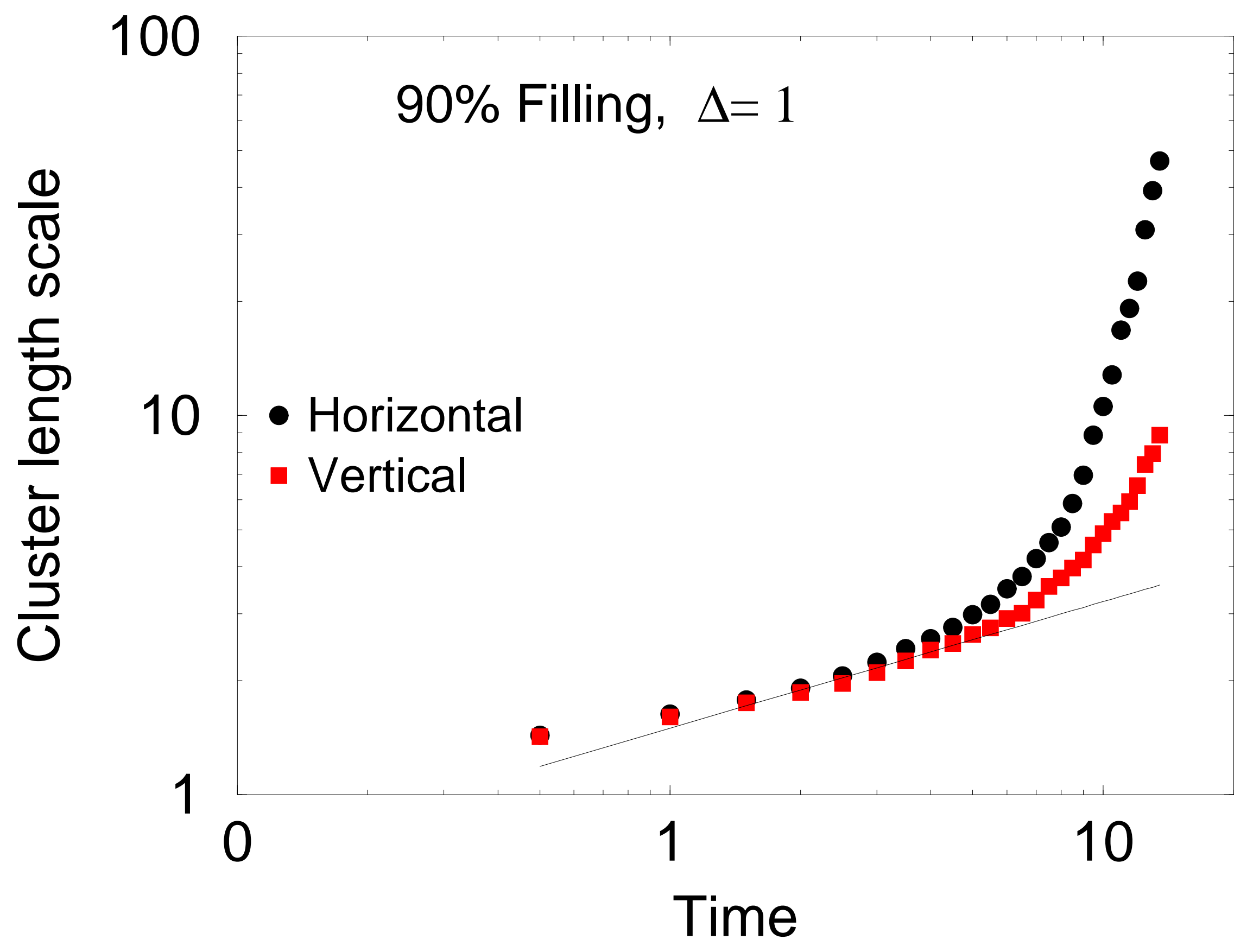




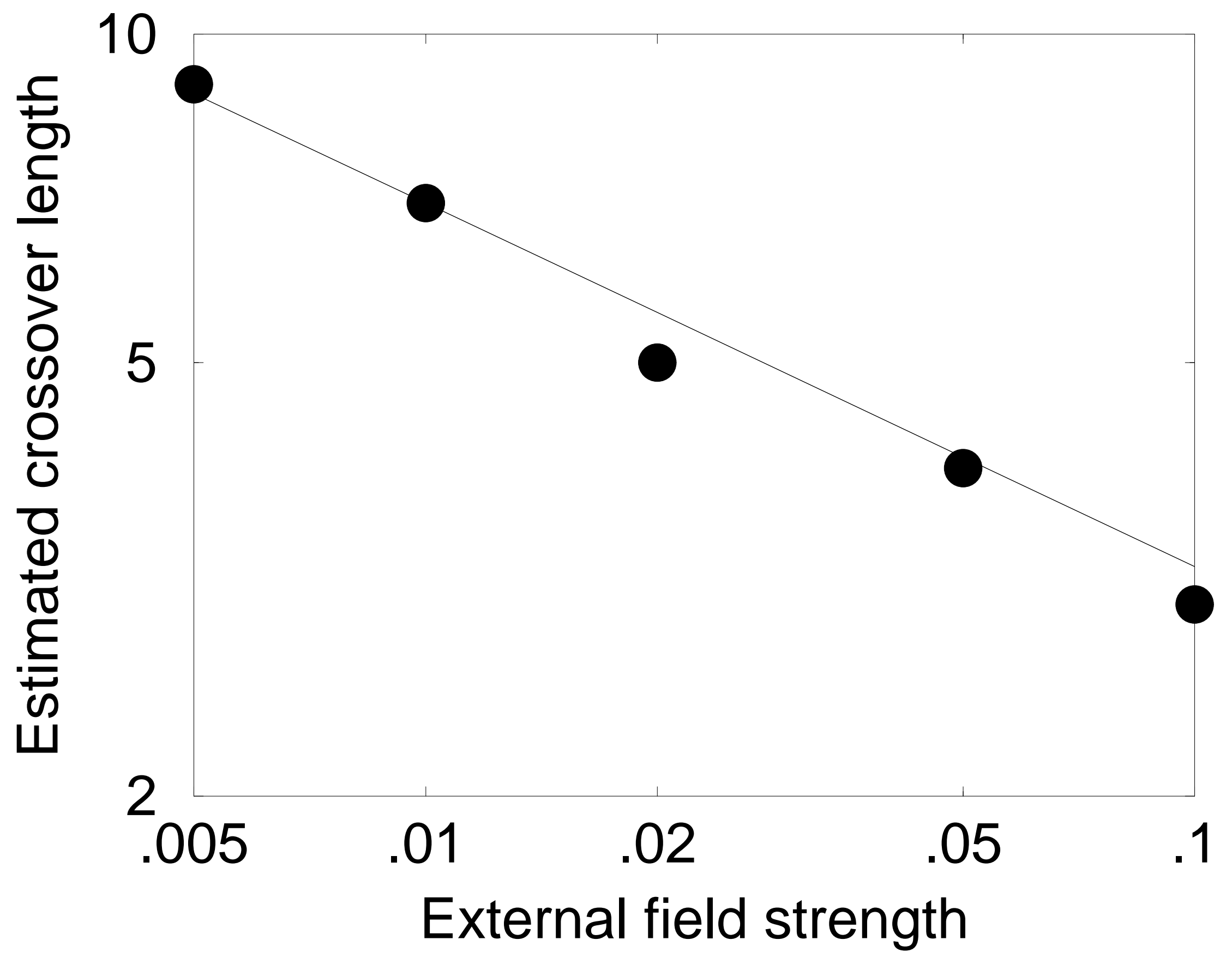




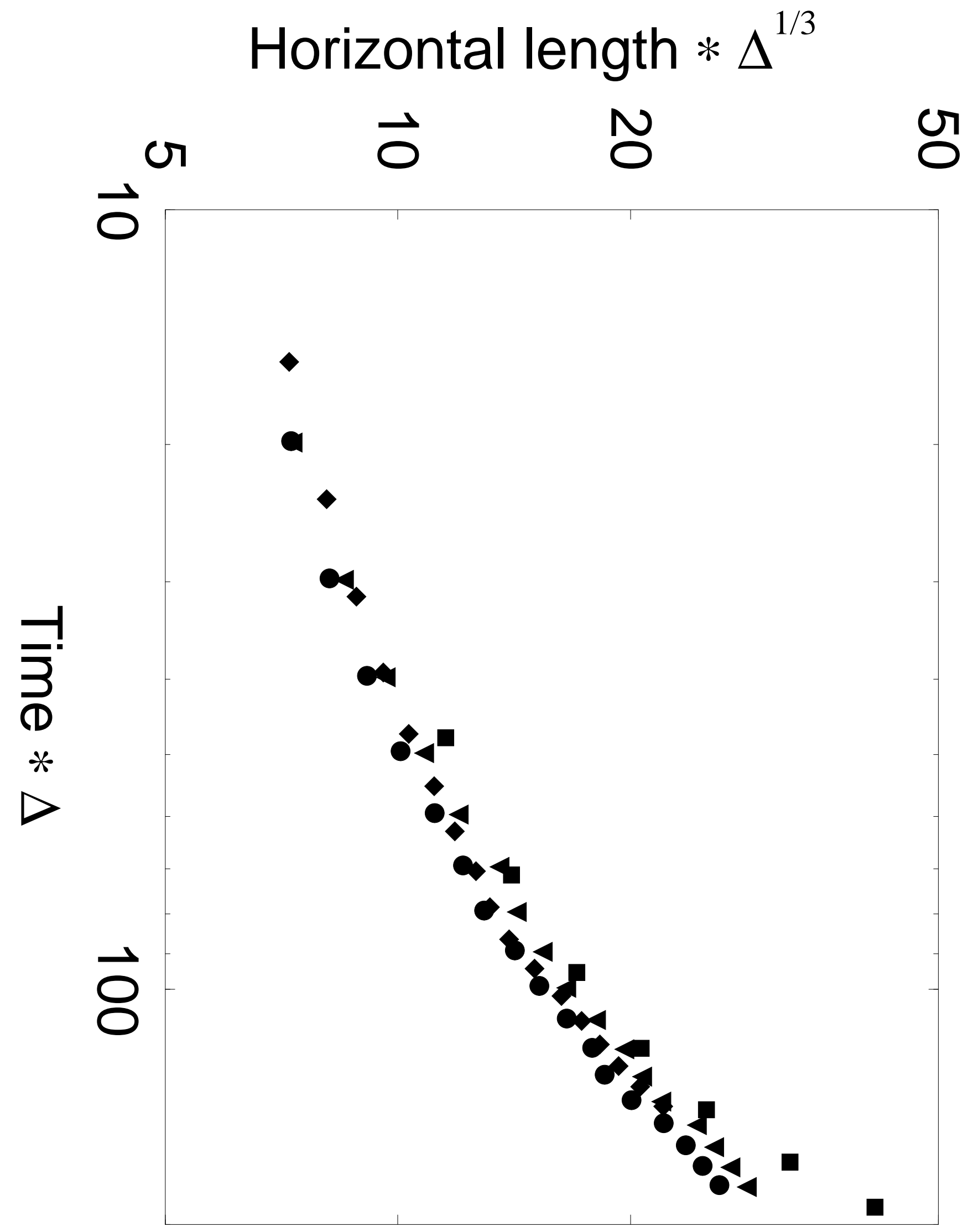

